\title{
Effects of Cooling Rate on the Microstructure of a Commercial Ni-Based Superalloy Using Atom Probe Tomography
}

\author{
P. M. Sarosi*, M. K. Miller**, D. Isheim***, M. J. Mills* \\ *The Ohio State University, 2041 College Rd, 477 Watts Hall, Columbus OH 43210. \\ **Oak Ridge National Laboratory, Oak Ridge, TN, 37831 \\ ***Northwestern University, Evanston, 2220 North Campus Drive, IL 60208
}

Nickel-base superalloys are widely used for high temperature structural materials such as hot sections of jet turbine engines because they possess the ability to retain excellent creep and yield strengths at high temperatures $\left(700^{\circ} \mathrm{C}\right)$. These strengths are derived from the distribution in the microstructure of the main constituent phases: $\gamma(\mathrm{Ni}-\mathrm{FCC})$ and $\gamma^{\prime}\left(\mathrm{L}_{2}\right.$-ordered structure based on $\mathrm{Cu}_{3} \mathrm{Au}$ ). More specifically, a bi-modal distribution of $\gamma^{\prime}$ provides the best combination of mechanical properties for commercial turbine disk alloys such as Rene 88 DT, and Rene 104 - the alloy in this investigation [1]. The importance of this type of microstructure was demonstrated [2] for a similar alloy, showing that just subtle changes in the smaller (tertiary) $\gamma^{\prime}$ distribution, resulting from different heat treatments, can profoundly improve the creep strength of disk alloys by inducing a remarkably sluggish creep deformation mechanism known as microtwinning [3]. This paper presents an examination of the effects of cooling rate on this and other microstructural parameters such as $\gamma / \gamma^{\prime}$ interface widths, element partitioning phase composition, crystal ordering and $\gamma^{\prime}$ precipitate size that also greatly impact the superalloy's creep strength using Atom Probe Tomography. The microstructures, of which a typical atom probe reconstruction is shown in Figure 1, from samples linearly cooled at different cooling rates were examined and compared. The $\gamma / \gamma^{\prime}$ interfacial widths, phase composition and element partitioning were obtained from proximity histograms [4] determined using Apex software [5].

The results showed that different microstructural parameters were affected differently by cooling rate. For example, the cooling rate significantly affected both secondary and tertiary $\gamma^{\prime}$ sizes, whereas the composition, volume fraction and element partitioning ratios were found to be insensitive to cooling rate. Furthermore, in the case of the interface width, cooling rate was found not to directly affect the $\gamma / \gamma^{\prime}$ interfacial width. Instead, faster cool resulted in the formation of smaller $\gamma^{\prime}$ precipitates, which possess wider $\gamma / \gamma^{\prime}$ interfacial widths than those of the larger precipitates. A typical proxigrams from the study is depicted in Figure 2. A full description of the proxigram analysis will be presented.

Along with the effects of cooling rate on microstructure the partial degree of order was also determined quantitatively using the compositional variation of the (100) planes in a $\gamma^{\prime}$ precipitate. The impact of crystal ordering on creep will then be discussed in the context of its effects on Complex Stacking Fault Energy.

In addition to the results described above, initial indications, also from the program analysis show that tungsten, segregates to the $\gamma / \gamma^{\prime}$ interface (Figure 2), which has not been observed previously in this alloy. The effect of $\mathrm{W}$ segregation on microstructure and thus creep is underway. 


\section{References}

[1] S.T Wlodeck, M Kelly, D. A. Alden Superalloys. Plymouth, New Hampshire: The Minerals, Metals \& Materials Society, 1996, pg 129.

[2] G.B Viswanathan, P.M. Sarosi, M.F Henry, D.D Whitis, W.W. Milligan W, M.J. Mills, Acta Materialia, 53 (2005) 53.

[3] M.Kolbe . Materials Science and Engineering A, 319-321 (2001) 383.

[4] O. Hellman, J. Vandenbroucke, J. Rusing, D Isheim, D.N Seidman. Microsc. and Microanal. 6 (2000) 437

[5] O. Hellman, Apex, Tomographic Softworks

[6] Research at the Oak Ridge National Laboratory SHaRE User Facility was sponsored by the Office of Basic Energy Sciences, Division of Scientific User Facilities, U.S. Department of Energy, under contract DE-AC05-00OR22725 with UT-Battelle, LLC.

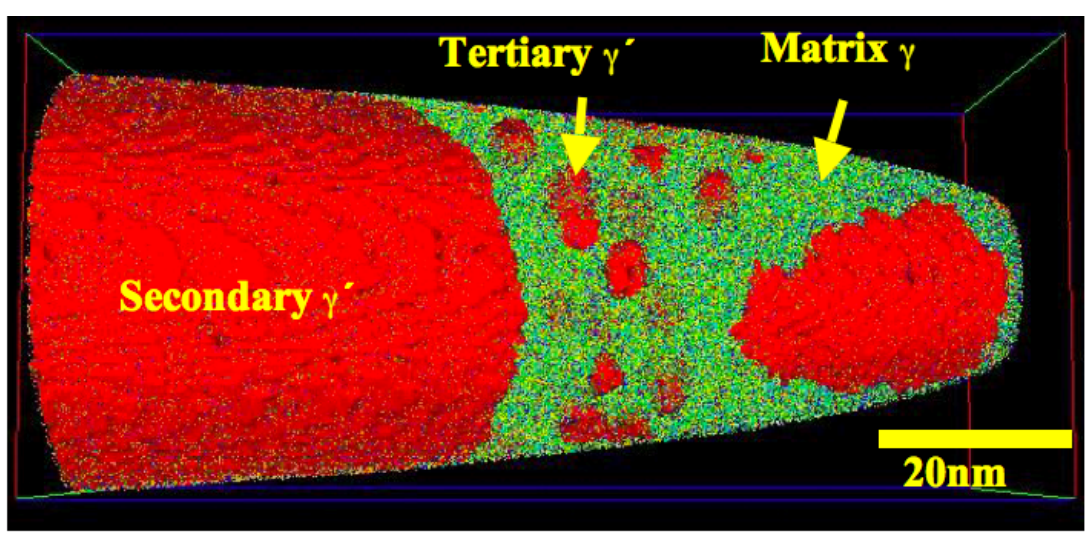

Figure 1: A typical 3D atom probe (3DAP) reconstruction of a bi-modal microstructure from Rene 104 with atoms (green) and a red isoconcentration surface delineating the $\gamma^{\prime}$ precipitates.
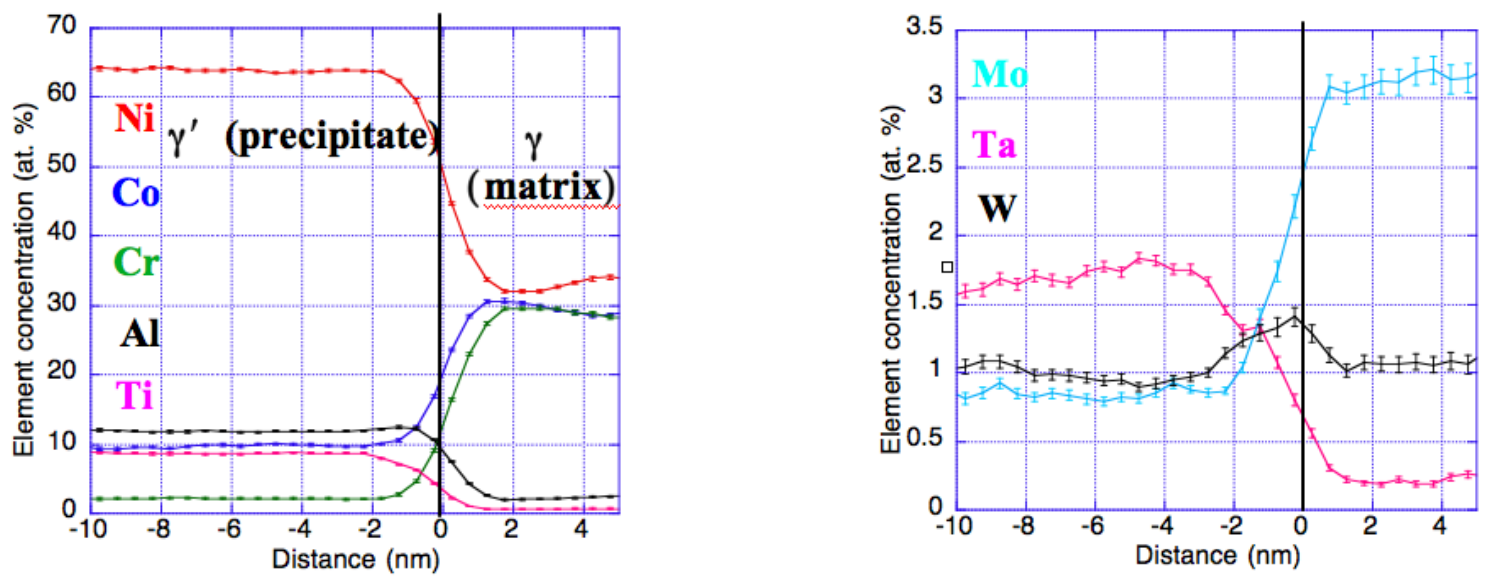

Figure 2: A typical proxigram of across the $\gamma / \gamma^{\prime}$ interface of secondary $\gamma^{\prime}$ precipitates from a sample cooled at $16.7^{\circ} \mathrm{C} / \mathrm{min}$, The $\gamma / \gamma^{\prime}$ interface is positioned at the $0 \mathrm{~nm}$ position with $\gamma^{\prime}$ at negative distances and $\gamma$ at positive distances. The segregation of tungsten (black line )at the interface is shown in the right hand proxigram. 